\title{
WT1, Monoclonal CEA, TTF1, and CA125 Antibodies in the Differential Diagnosis of Lung, Breast, and Ovarian Adenocarcinomas in Serous Effusions
}

\author{
Weijian Zhu, M.D., Ph.D. and Claire W. Michael, M.D. ${ }^{\star}$
}

The distinction between metastatic adenocarcinomas of lung $(L A C)$, breast $(B A C)$, and ovary $(O A C)$ in serous effusions can be very difficult since they all can present as tight cell clusters. This is particularly challenging when the malignant effusion is the patient's initial presentation or when the patient has a history of more than one primary. The aim of this study is to evaluate the usefulness of WT1, monoclonal CEA ( $m C E A), T T F 1$, and CA125 antibodies in the differential diagnosis of metastatic adenocarcinoma from the lung, breast and ovary in serous effusions.

Forty-six samples of serous effusions with their corresponding cell blocks were retrieved from our hospital computer system, including 13 BACs, 13 LACs, and 20 OACs. The diagnoses were confirmed by the surgical resection. Formalin-fixed and paraffinembedded cell block sections were immunostained for WTI, $m C E A, T T F 1$, and CA125. Two observers blindly reviewed the immunostained slides without knowledge of the previous clinical or histologic diagnoses. The staining intensity was graded semiquantitatively as negative, 0; weak, 1+; moderate, 2+; and strong, $3+$. The percentage of positively staining cells was estimated. The distribution patterns of reactivity for WTI and TTFI were recorded as nuclear, and $m C E A$ and CA125 as membranous stain.

Metastatic OACs showed positive immunoreactivity to WT1 in $19 / 20$ (95\%) cases, CA125 in 20/20 (100\%), and all showed negative reaction for both $m C E A(0 / 20,0 \%)$ and TTF 1 (0/20, $0 \%)$. BAC showed positive reaction in $6 / 13$ (46\%) cases to

Department of Pathology, University of Michigan Hospitals, Ann Arbor, Michigan

*Correspondence to: Claire W. Michael, M.D., Department of Pathology, University of Michigan Hospitals, 1500 E. Medical Center Drive, Room 2G332 University Hospital, Ann Arbor, MI 48109-0054.

E-mail: clairemi@med.umich.edu

Received 13 June 2006; Accepted 22 March 2007

DOI 10.1002/dc.20643

Published online in Wiley InterScience (www.interscience.wiley.com).
CA125 and mCEA. Staining pattern was diffuse for CA125 and focal for $m C E A$. Only 2/13 (15\%) were positive for WT1, while all of 13 BAC cases $(0 / 13,0 \%)$ were negative for TTF1. LAC showed positive immunoreactivity for TTF1 in 9/13 (69\%) with a characteristic nuclear staining pattern, but only 3/13 (23\%) were focally stained for WT1. In addition, 8/13 (62\%) of LAC cases were positive for both CA125 and $m C E A$.

Our results demonstrate that the WT1 stain is specific for metastatic carcinoma of ovarian primary, showing a high sensitivity. In addition, CA125 stain is very sensitive for OACs, but could be positive in about a half of LAC and BAC cases. An immunostaining pattern of positive $m C E A$ as well as negative WT1 rules out OACs, raising the possibility of LACs and BACs. A positive TTFI staining supports the diagnosis of metastatic carcinoma originating from lung rather than breast, while a negative TTF 1 favors the diagnosis of a breast primary. Immunohistochemical studies with WT1, TTF1, and mCEA antibodies are useful in the differential diagnosis of metastatic adenocarcinomas of lung, breast, and ovary. Diagn. Cytopathol. 2007; 35:370-375. o 2007 Wiley-Liss, Inc.

Key Words: immunostaining; serous effusion; adenocarcinoma; WT1, CA125, TTF1 and mCEA

\section{Introduction}

Malignant serous effusion is not an uncommon clinical manifestation of adenocarcinoma. ${ }^{1,2}$ The distinction between metastatic adenocarcinomas of lung (LAC), breast (BAC), and ovary (OAC) in serous effusions could be very difficult or sometimes impossible on the basis of morphology alone. ${ }^{1-3}$ This is particularly challenging when the malignant effusion is the patient's initial presentation or when the patient has a history of more than one primary. However, elucidating the origin of these malignant neoplasms often may have therapeutic consequences 
for the patient. ${ }^{4}$ Many antibodies directed against specific cell type antigens have been used in LAC, BAC, and $\mathrm{OAC}$ on the histological section and/or serous effusions to enhance the pathological diagnosis, with varying degrees of efficacy but the optimum panel of antibodies is yet to be reported. When used in specific panels, immunohistochemical studies can be extremely useful for determining tumor type, particularly in cases with an unknown primary site. ${ }^{5-8}$

WT1, a tumor suppressor gene initially identified as contributing to the development of Wilms tumor, is expressed preferentially in the urogenital system and mesoderm-derived tissues. ${ }^{9,10}$ Relatively specific reactivity for WT1 protein has been shown in ovarian papillary serous carcinoma and in a number of different carcinomas in addition to mesothelioma, desmoplastic small round cell tumor, and Wilms tumor. ${ }^{11-13}$

There are no specific markers of ovarian adenocarcinoma, one of the most common neoplasms to present with ascites. Antibodies such as CA125 and human alveolar macrophage (HAM) 56 have been proposed as useful markers of ovarian adenocarcinoma, but immunoreactivity is often present in adenocarcinomas of other sites, limiting their diagnostic value. ${ }^{12,14,15}$

Thyroid transcription factor1 (TTF-1) has been described as a marker that reportedly recognizes carcinomas of pulmonary origin, particularly pulmonary adenocarcinoma. ${ }^{16-18}$ TTF-1 is also expressed in the thyroid gland, the diencephalon, and the bronchioalveolar epithelium. ${ }^{18-21}$

Monoclonal carcinoembryonic antigens (mCEA) are of value in confirmation of an adenocarcinoma, as they are commonly positive in these neoplasms but rarely so in reactive mesothelial cells or mesothelioma. ${ }^{15,22}$ However, certain adenocarcinomas, especially ovarian serous adenocarcinomas, are characteristically negative with mCEA. ${ }^{15}$

The aim of this study was to evaluate the usefulness of WT1, monoclonal CEA (mCEA), TTF1, and CA125 antibodies as markers in the differential diagnosis of ovarian, lung, and breast metastatic adenocarcinomas in cytologic material prepared from malignant effusion specimens.

\section{Materials and Methods}

A total of 46 cases of serous effusions were retrieved from our hospital computer system, including $13 \mathrm{BACs}$, 13 LACs, and 20 OACs. The diagnosis of each case was confirmed by surgical excision and histologic examination of the primary tumor. Formalin-fixed and paraffin-embedded cell block sections from the serous effusions were stained with hematoxylin and eosin to evaluate the presence of tumor cells in each cell block. Immunohistochemical staining was performed on the DAKO Autostainer (DAKO, Carpinteria, CA) using DAKO LSAB + and 3,3'diaminobenzidine as the chromogen. Deparaffinized sec-
Table I. Immunoreactivity for WT1, CA125, TTF1 and mCEA in Malignant Effusions of Ovary, Lung, and Breast Adenocarcinomas

\begin{tabular}{lccccc}
\hline & & \multicolumn{4}{c}{$\begin{array}{c}\text { No. of cases immunoreactive } \\
\text { for various markers }\end{array}$} \\
\cline { 3 - 6 } Primary sites of & $\begin{array}{c}\text { No. of } \\
\text { cases }\end{array}$ & $\begin{array}{c}W T 1 \\
(\%)\end{array}$ & $\begin{array}{c}\text { CA125 } \\
(\%)\end{array}$ & $\begin{array}{c}\text { TTF1 } \\
(\%)\end{array}$ & $\begin{array}{c}\text { mCEA } \\
(\%)\end{array}$ \\
\hline Ovary & 20 & $19(95)$ & $20(100)$ & $0(0)$ & $0(0)$ \\
Lung & 13 & $3(23)$ & $8(62)$ & $9(69)$ & $8(62)$ \\
Breast & 13 & $2(15)$ & $6(46)$ & $0(0)$ & $6(46)$ \\
\hline
\end{tabular}

tions of formalin-fixed tissue at $4-\mu \mathrm{m}$ thickness were stained with H\&E or labeled with anti-WT1 (1:50; Cell Marque, Hot Springs, AR), anti-TTF1 (1:200; DAKO Cytomation, Carpinteria, CA), anti-mCEA (1:1; Cell Marque), and anti-CA125 (1:100; Vector Laboratories, Newcastle upon Tyne, UK) along with positive and negative controls. Citrate antigen retrieval (20 min) was used before incubation with antibodies. An appropriate positive control slide for each marker evaluated was included in each run. Control slides substituting tris buffer for primary antibody were run as negative controls. Two observers (WZ \& CWM) blindly reviewed the immunostaining without knowledge of the previous clinical or histopathologic diagnoses. At least 200 cells were counted and the extent of staining was assessed as percentage of neoplastic cells, and intensity of staining was graded on a 0 to $3+$ scale semiquantitatively (negative, 0 ; weak, $1+$; moderate, $2+$; strong, $3+$ ). The distribution pattern of immunoreactivity for WT1 and TTF1 was recorded as nuclear, and mCEA and CA125 as membranous staining.

\section{Results}

Immunoreactivity for WT1, CA125, TTF1, and mCEA was studied in a total of 46 cases of metastatic adenocarcinoma in cell blocks prepared from serous effusions, including 13 BACs, 13 LACs, and 20 OACs. Overall results are summarized in Table I.

\section{WT1}

WT1 immunoreactivity was detected in 19/20 (95\%) OCA cases, exhibiting an immunoreactivity in at least $56.6 \%$ of neoplastic cells (Fig. C-1). In comparison, only $3 / 13(23 \%)$ LAC and 2/13 BAC (15\%) cases displayed a WT1 immunoreactivity in $40.7 \%$ and $40.0 \%$ of neoplastic cells, respectively. WT1 immunoreactivity demonstrated a strong nuclear staining pattern, showing an average intensity of 2.67 in LCA, 2.58 in OAC, and 2.5 in BAC neoplastic cells.

\section{CA125}

CA125 immunoreactivity was detected in 20/20 (100\%) OAC cases, demonstrating the characteristic membranous staining pattern in $92.3 \%$ of neoplastic cells, rimming 


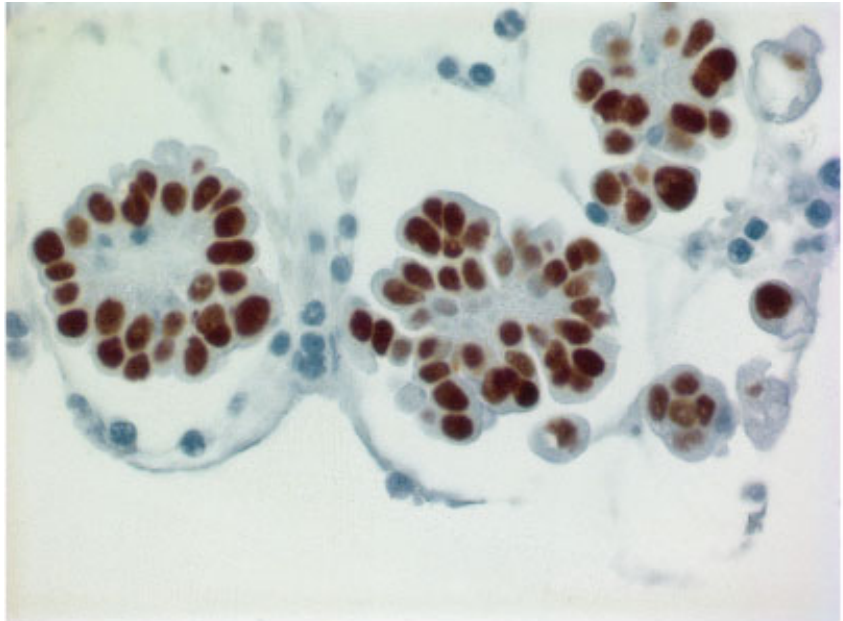

Fig. C-1

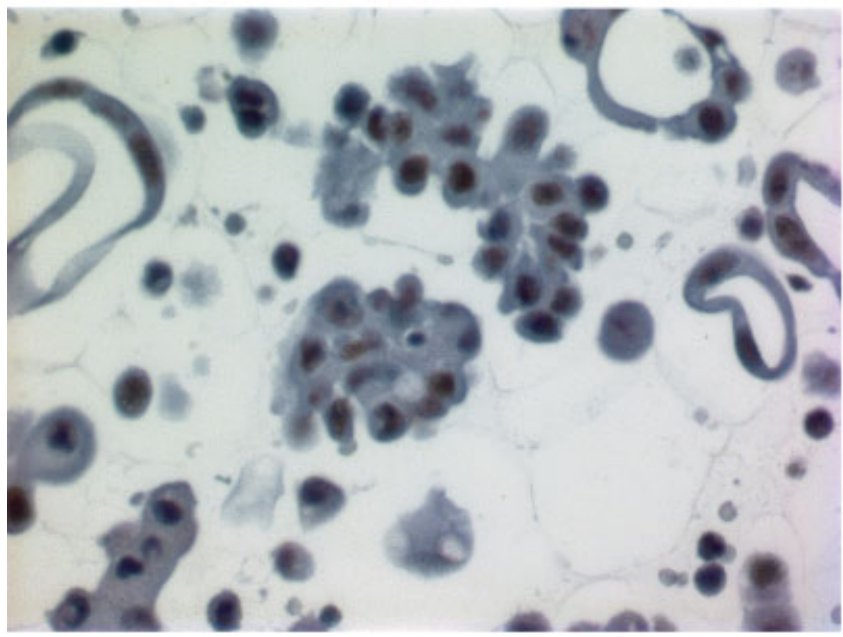

Fig. C-3

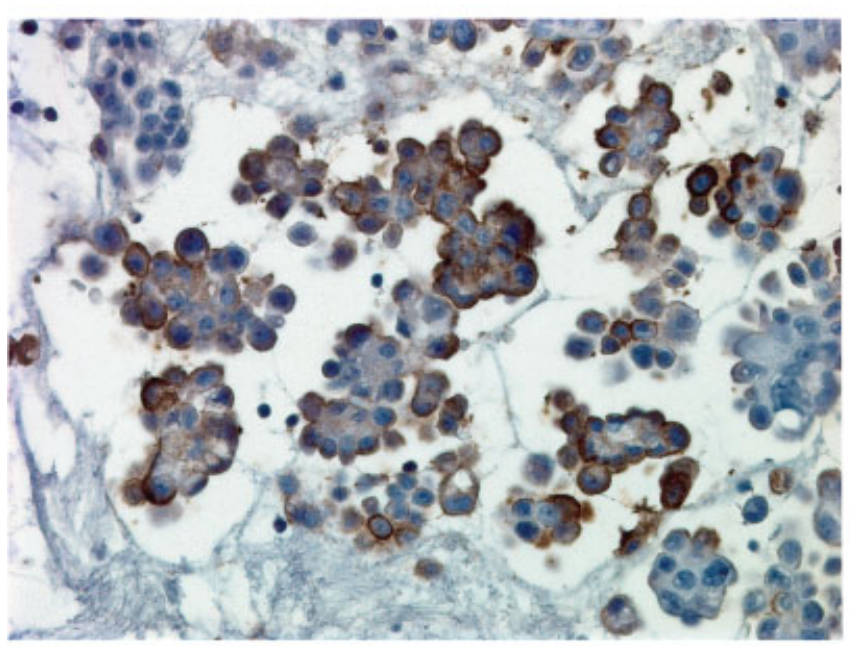

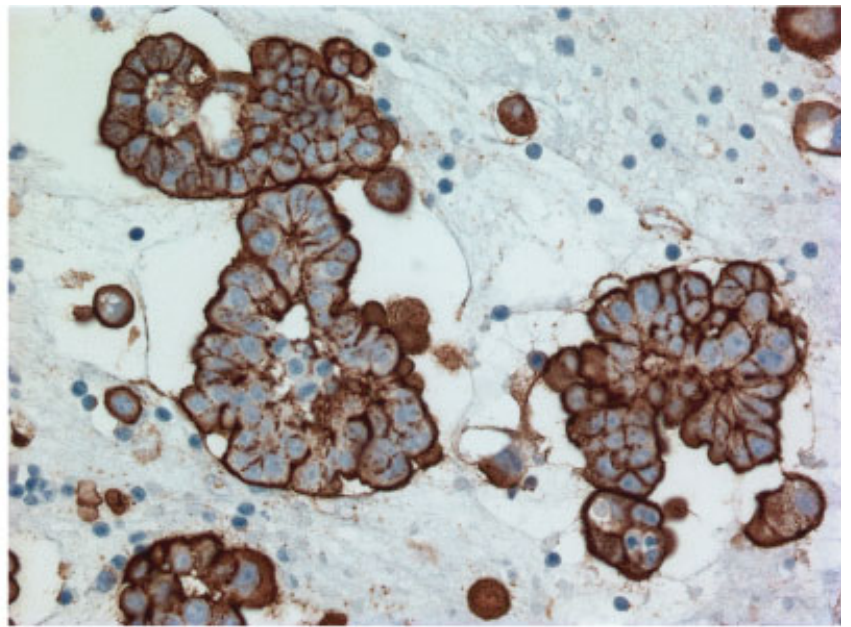

Fig. C-2

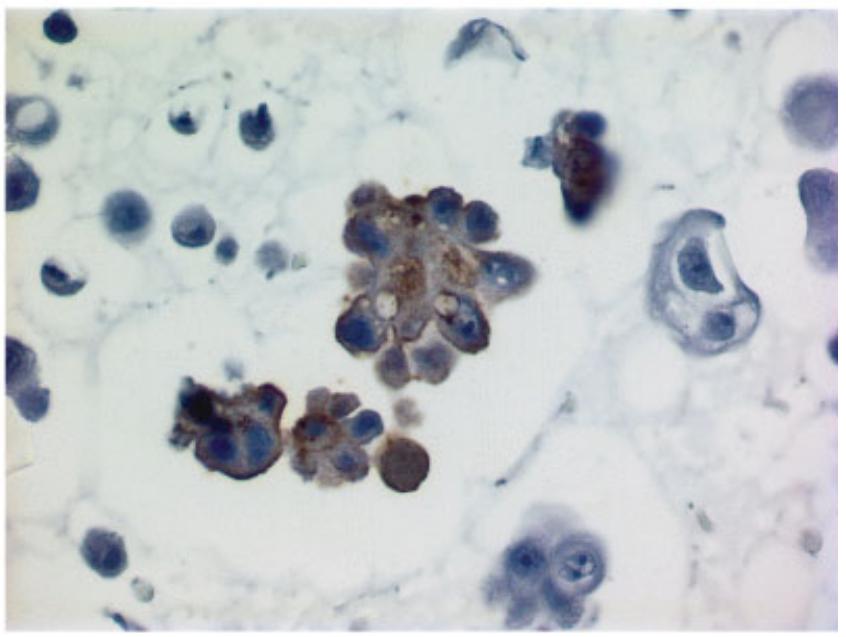

Fig. C-4

Fig. C-5

Figs. C-1-C-5. Fig. C-1. Strong nuclear immunoreaction for WT-1 in a metastatic ovarian adenocarcinoma, $\times 600$. Fig. C-2. Strong membranous immunoreaction to CA125 in a metastatic ovarian adenocarcinoma, $\times 400$. Fig. C-3. Moderate nuclear immunoreaction to TTF-1 in a metastatic lung adenocarcinoma, $\times 600$. Fig. C-4. Moderate membranous staining to mCEA detected in metastatic lung adenocarcinoma, $\times 600$. Fig. C-5. Moderate membranous immunoreaction to mCEA in a metastatic breast adenocarcinoma, $\times 400$. 


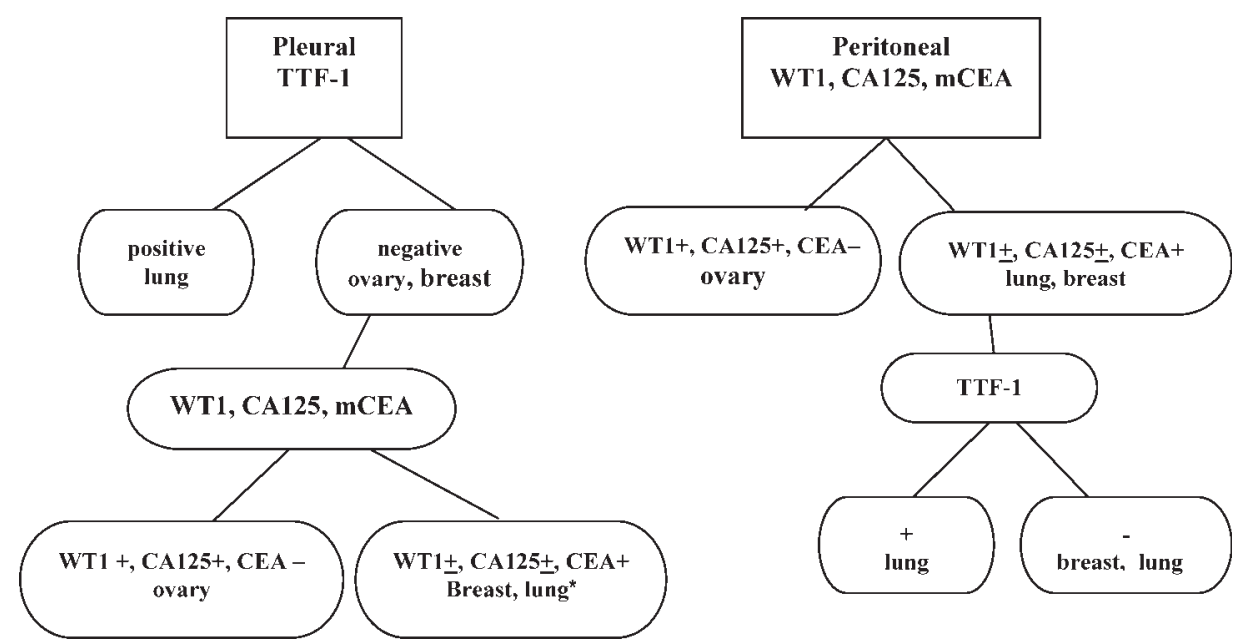

Fig. 1. Proposed application of antibody panel in the differential diagnosis of ovarian, lung, and breast adenocarcinoma. Asterisk indicates that lung adenocarcinoma usually reacts more diffusely with CA125, while breast tends to be focally reactive.

around the tumor cell aggregates (Fig. C-2). CA125 was positive in 8/13 (62\%) LAC and 6/13 (46\%) BAC cases, with a similar staining pattern as the tumor cells in ovary. In the positively staining lung and breast cases, about $70.6 \%$ and $90 \%$ neoplastic cells were stained with CA125, respectively. Although OAC showed a uniformly 3+ staining, there is no significant difference in the staining intensity in comparison with LAC (2.88) and BAC (2.83) cases.

\section{TTF 1}

TTF1 immunoreactivity was detected in 9/13 (69\%) LAC with the characteristic nuclear staining pattern and an average of 2.0 intensity (Fig. C-3). No nuclear immunostaining was detected in either the $\mathrm{OAC}(0 / 20,0 \%)$ or BAC cases $(0 / 13,0 \%)$.

\section{$m C E A$}

mCEA immunoreactivity with a membranous staining pattern was detected in 8/13 (62\%) LAC and in 6/13 (46\%) BAC cases (Figs. C-4 and C-5). The average staining intensity was 2.75 for both LAC and 2.67 for BAC. In contrast, there is negative immunoreaction with mCEA for all 20 OAC cases.

\section{Discussion}

Serous effusions may be the first symptom of both mesotheliomas and metastatic adenocarcinomas. In these circumstances, cytology can play a critical role in the diagnosis. The initial challenge is to distinguish the malignant mesothelioma (MM) from metastatic adenocarcinoma in cytologic specimens, which is aided greatly by the use of a panel of antibody immunocytochemistry. ${ }^{1-8}$ The next challenge is to identify the primary neoplasm in the case of adenocarcinoma manifesting initially as an effusion or if the patient has a history of more than one primary. In this study, we evaluated the usefulness of WT1, monoclonal CEA (mCEA), TTF1, and CA125 antibodies in the differential diagnosis of metastatic LAC, BAC, and OAC in serous effusions.

WT1 is a tumor suppressor gene implicated in the development of Wilms tumor and has been demonstrated in a high percentage of OAC and in some BAC. ${ }^{1-13}$ The frequent expression of WT1 in ovarian serous and transitional cell carcinomas, and its infrequent expression in other ovarian carcinoma subtypes, such as clear cell, mucinous, and endometrioid adenocarcinomas, has been well documented. ${ }^{10}$ Our study demonstrates that WT-1 is a highly sensitive marker of OAC and its expression is only seen in a small fraction of lung and breast carcinomas (23 and 15\%, respectively). However, there was no significant difference noted in the intensity and the percentage of stained neoplastic cells in any of the immunoreactive BAC and LAC cases when compared with OAC. These results are in agreement with previous studies. ${ }^{9-13,15,18}$ In addition to being a sensitive marker of ovarian serous carcinomas in the workup of carcinoma of unknown primary, WT-1 is also a relatively specific ovarian marker. We believe that WT-1 should be included in the immunostaining panel of malignant effusions of unknown primary and that a negative reaction would argue strongly against the diagnosis of OAC.

Overall, $69 \%$ of primary pulmonary adenocarcinomas showed staining for TTF-1, and no immunoreactivity was noted in ovarian and breast carcinomas. TTF1 immunostaining was characterized by a nuclear pattern in $69 \%$ of tumor 
cells, with a moderate intensity (2.0). Our data are very close to the findings of several previous reports, where the range of positive reaction was $72-80 \%$, and is also consistent with the almost universal finding that anti-TTF-1 only stains adenocarcinomas that are primary in the lung and thyroid gland. ${ }^{16-22}$ The usefulness of TTF-1 as a marker of pulmonary adenocarcinoma diagnosis is confirmed by our study, although it is limited by the sensitivity of TTF1 staining. ${ }^{16,17}$ TTF-1 is the best marker to confirm the diagnosis of LAC; however, a negative TTF-1 expression does not rule out a lung primary and a panel of antibodies will be an important part in determining the primary site.

CEA is a glycoprotein within a large family of glycoproteins, which is expressed in adenocarcinomas in a variable percentage, depending on the monoclonal or polyclonal antibodies. The percentage of carcinomas positive for CEA is variable, depending on the primary site and the subtype of carcinoma. ${ }^{15,22}$ Our study demonstrated that a membranous mCEA expression pattern was found in 8/12 (67\%) LAC and 6/13 (46\%) BAC, but no mCEA immunoreactivity was detected in OAC. This finding supports the value of monoclonal carcinoembryonic antigen (mCEA) in the differential diagnosis as it is commonly positive in breast and lung carcinomas, while characteristically negative in ovarian serous adenocarcinoma. Therefore, CEA is one of the best negative antibodies for $\mathrm{OAC}$, and a positive expression of mCEA in the metastatic neoplastic cells argues against such diagnosis. It is worth noting that this stands true only for the serous type and that mucinous ovarian adenocarcinomas can be positive for mCEA and negative for WT-1. In addition, our findings revealed that there is a much higher expression of mCEA in LAC than BAC.

To date, there are no specific markers of ovarian adenocarcinoma which is one of the most common neoplasms to present with ascites. ${ }^{11,12} \mathrm{CA} 125$ is a glycoprotein identified on the cell membrane in celomic epithelium during embryogenesis, expressed in a high percentage of ovarian carcinomas. More than $90 \%$ of ovarian cancers and 10-30\% of primary breast cancers have been reported to express CA125. ${ }^{15}$ The expression of CA125 has also been detected in tissue sections of numerous other tumors, including those of lung, stomach, pancreas, and mesothelium, limiting its diagnostic value. ${ }^{12-15}$ Our study showed that CA125 was expressed in 20/20 OCA cases (100\%) with a characteristic membranous staining pattern, rimming around the tumor cell aggregates as compared with $8 / 13(61.5 \%)$ of metastatic lung cancers and 6/13 (46\%) of metastatic breast cancers. CA125 demonstrates a typically strong and diffuse pattern in metastatic ovary and breast carcinomas, in contrast with a relatively focal staining pattern in the metastatic lung carcinoma.

In conclusion, positive immunoreactivity for WT1 and/ or CA125 with a negative reaction to CEA would be sup- portive of an OAC particularly in a peritoneal fluid. A positive CEA argues against the diagnosis of OAC of the serous type. In the event of a positive CEA, TTF- 1 would be useful in confirming a LAC particularly in a pleural fluid (Fig. 1). BAC is less likely to express CEA and WT-1 than LAC.

\section{References}

1. Hammar SP. Metastatic adenocarcinoma of unknown primary origin. Hum Pathol 1998;29:1393-1402.

2. Nystrom JS, Weiner JM, Heffelfinger-Juttner J, Irwin LE, Bateman JR, Wolf RM. Metastatic and histologic presentations in unknown primary cancer. Semin Oncol 1977;4:53-58.

3. Varadhachary GR, Abbruzzese JL, Lenzi R. Diagnostic strategies for unknown primary cancer. Cancer 2004;100:1776-1785.

4. Pavlidis N, Briasoulis E, Hainsworth J, Greco FA. Diagnostic and therapeutic management of cancer of an unknown primary. Eur $\mathrm{J}$ Cancer 2003;39:1990-2005.

5. Brown RW, Campagna LB, Dunn JK, Cagle PT. Immunohistochemical identification of tumor markers in metastatic adenocarcinoma.A diagnostic adjunct in the determination of primary site. Am J Clin Pathol 1997;107:12-19.

6. Pecciarini L, Giulia Cangi M, Doglioni C. Identifying the primary sites of metastatic carcinoma: the increasing role of immunohistochemistry. Curr Diagn Pathol 2001;7:168-175.

7. Moch H, Oberholzer M, Dalquen P, Wegmann W, Gudat F. Diagnostic tools for differentiating between pleural mesothelioma and lung adenocarcinoma in paraffin embedded tissue. Part I: Immunohistochemical findings. Virchows Arch A Pathol Anat Histopathol 1993;423:19-27.

8. Skov B, Lauritzen A, Hirsch F, Skov T, Nielsen H. Differentiation of adenocarcinoma of the lung and malignant mesothelioma: Predictive value and reproducibility of immunoreactive antibodies. Histopathology 1994;25:431-437.

9. Ordonez NG. The immunohistochemical diagnosis of mesothelioma. A comparative study of epithelioid mesothelioma and lung adenocarcinoma. Am J Surg Pathol 2003;27:1031-1051.

10. Logani S, Oliva E, Amin MB, Folpe AL, Cohen C, Young RH. Immunoprofile of ovarian tumors with putative transitional cell (urothelial) differentiation using novel urothelial markers: Histogenetic and diagnostic implications. Am J Surg Pathol 2003;27(11): 1434-1441.

11. Lagendijk JH, Mullink H, van Diest PJ, Meijer GA, Meijer CJ. Immunohistochemical differentiation between primary adenocarcinomas of the ovary and ovarian metastases of colonic and breast origin. Comparison between a statistical and an intuitive approach. J Clin Pathol 1999;52:283-290.

12. Tornos C, Soslow R, Chen S, et al. Expression of WT1, CA 125, and GCDFP-15 as useful markers in the differential diagnosis of primary ovarian carcinomas versus metastatic breast cancer to the ovary. Am J Surg Pathol 2005;29(11):1482-1489.

13. Lee BH, Hecht JL, Pinkus JL, Pinkus GS. WT1, estrogen receptor, and progesterone receptor as markers for breast or ovarian primary sites in metastatic adenocarcinoma to body fluids. Am J Clin Pathol 2002; 117(5):745-750.

14. Loy TS, Quesenberry JT, Sharp SC. Distribution of CA125 in adenocarcinomas: An immunohistochemical study of 481 cases. Am J Clin Pathol 1992;98:175-179.

15. Tornos C, Soslow R, Chen S, et al. Expression of WT1, CA 125, and GCDFP-15 as useful markers in the differential diagnosis of primary ovarian carcinomas versus metastatic breast cancer to the ovary. Am J Surg Pathol 2005;205(29):1482-1489.

16. Stenhouse G, Fyfe N, King G, Chapman A, Kerr KM. Thyroid transcription factor 1 in pulmonary adenocarcinoma. J Clin Pathol 2004; 57(4):383-387. 
17. Wu M, Szporn AH, Zhang D, et al. Cytology applications of p63 and TTF-1 immunostaining in differential diagnosis of lung cancers. Diagn Cytopathol 2005;33(4):223-227.

18. Kaufmann O, Dietel M. Thyroid transcription factor-1 is the superior immunohistochemical marker for pulmonary adenocarcinomas and large cell carcinomas compared to surfactant proteins $\mathrm{A}$ and $\mathrm{B}$. Histopathology 2000;36(1):8-16.

19. Bejarano PA, Nikiforov YE, Swenson ES, Biddinger PW. Thyroid transcription factor-1, thyroglobulin, cytokeratin 7, and cytokeratin 20 in thyroid neoplasms. Appl Immunohistochem Mol Morphol 2000;8(3):189-194.
20. Miettinen M, Franssila KO. Variable expression of keratins and nearly uniform lack of thyroid transcription factor 1 in thyroid anaplastic carcinoma. Hum Pathol 2000;31(9):1139-1145.

21. Zhang $P$, Zuo H, Nakamura $Y$, Nakamura $M$, Wakasa $T$, Kakudo K. Immunohistochemical analysis of thyroid-specific transcription factors in thyroid tumors. Pathol Int 2006;56(5): 240-245.

22. Rishi M, Kovatich A, Ehya H. Utility of polyclonal and monoclonal antibodies against carcinoembryonic antigen in hepatic fine-needle aspirates. Diagn Cytopathol 1994;11(4):358-361. Discussion 361362. 